\title{
Note About Non-BPS and BPS Dp-branes in Near Horizon Region of $N$ Dk-Branes
}

\author{
by J. Klusoň \\ Department of Theoretical Physics and Astrophysics \\ Faculty of Science, Masaryk University \\ Kotlárská 2, 611 3\%, Brno \\ Czech Republic \\ E-mail: klu@physics.muni.cz
}

\begin{abstract}
In this paper we will consider the dynamics of BPS and non-BPS Dp-branes in the background of $N$ Dk-branes. Our approach is based on an existence of the new symmetry of D-brane effective actions that naturally emerges in the near horizon region of the stack of $N$ Dk-branes. Since generally this scaling symmetry is explicitly broken in the Lagrangian we will find the equation that determines the time evolution of the generator of this transformations. Then we will argue that in case when the tachyon living on the worldvolume of unstable D-brane reaches the stable minimum the time evolution of this generator can be easily determined. With the help of the knowledge of the time dependence of this charge we will determine the trajectory of the non-BPS D-brane in the near horizon region of $N$ Dk-branes. In case of BPS Dp-brane probe we will aruge that such a broken scaling symmetry exists as well and the existence of the explicit time dependence of the generator of this symmetry can be used in the solving the equation of motion of the probe Dp-brane in the near horizon region of $N$ Dk-branes.
\end{abstract}

KeYwords: D-branes. 


\section{Contents}

1. Introduction 1

2. The effective action for non-BPS Dp-brane in Dk-brane background 2

3. Non-BPS Dp-brane in its stable vacuum 7

4. Dynamics of the BPS Dp-brane in the near horizon region of $N$ Dk-branes and broken scaling symmetry 10

5. Conclusion 14

\section{Introduction}

The study of unstable objects in string theory ${ }^{1}$ led to the deep insight into the string theory. As is well know the decay of unstable D-brane is triggered by rolling the open string tachyon [1, 2, 3]. An interesting consequence of this research is the fact that the effective field theory description of the tachyon condensation [20, 21, 22, 23] is very powerful even if the tachyon is of order of the string scale. This issue was extensively discussed in [24, 25, 27, 26].

A situation in which dynamics involved is quite analogous to the tachyon rolling is the formation of non-threshold bound states. A particular example of such a process is falling probe BPS D-brane into a target stack of $N$ NS5-branes [5] ${ }^{2}$. Interestingly, the dynamics of the rolling of the radial mode("radion") resembles tachyon rolling dynamics of unstable D-brane. In particular, for appropriate background and regime of target NS5-branes or Dk-branes the works cited above noted that the radion effective action takes exactly the same functional form as the tachyon effective action for unstable D-brane. The natural proposal is then to view the radion rolling dynamics as a sort of "geometrical realisation" of the tachyon rolling dynamics for unstable D-brane.

The extension of these works to the case of a non-BPS Dp-brane probe has been done in [10, 11, 19]. Generally the study of the dynamics of non-BPS Dp-brane is much more involved than in case of BPS Dp-brane thanks to the presence of the

\footnotetext{
${ }^{1}$ For recent review that also contains extensive list of references, see 4$]$.

${ }^{2}$ Related problems were also studied in [6, 7, 8, 9, 12, 13, 14, 15, 16, 17, 18, 19.
} 
tachyon on the worldvolume of non-BPS Dp-brane. However we have argued in [10, 19] that in region of the tachyon and radion field theory space where tachyon is large and Dp-brane is in the near horizon region of $N$ Dk-branes of NS5-branes there exists additional worldvolume symmetry ${ }^{3}$ of the action that simplifies the analysis of the resulting equations of motion and we were able to study the time evolution of the radion and tachyon on the worldvolume of a non-BPS Dp-brane. It is however important to stress that the existence of this symmetry was restricted to the case of the motion of Dp-brane in the background of $N$ NS5-branes or in the background of $N$ D3-branes.

In this paper we will continue further the study of the properties of this new symmetry (possible explicitly broken) of the tachyon effective action in the Dkbrane background. We will find that the tachyon effective action in the near horizon region and for large tachyon is invariant under the form of scaling transformation introduced bellow on condition that the parameter $\lambda \equiv N g_{s} l_{s}^{7-k}$, where $N$ is a number of background Dk-branes, $l_{s}$ is the string length and $g_{s}$ is string coupling, transforms under these transformations as well. We will determine the generator of the scaling symmetry from the tachyon effective action and then we find the equation that governs the time evolution of the generator $D^{4}$. It turns out however that for the tachyon that reaches its stable minimum this equation takes particular simple form that allows us to find the explicit time dependence of $D$. Then using this fact we will solve the equations of motion for $R$ and hence we will able to determine the time evolution of the non-BPS Dp-brane in the near horizon region of $N$ Dk-branes in situation when $T$ reaches its stable minimum.

The paper is organised as follows. In the next section (2) we introduce the form of the tachyon effective action in the near horizon region of $N$ Dk-branes. Then we will study the symmetries of this action using careful application of the Noether theorem. In section (3) we will study the time evolution of the non-BPS Dp-brane where the worldvolume tachyon is in its stable minimum. In section (4) we will study the dynamics of a BPS Dp-brane in the background of $N$ Dk-branes that is based on an existence of the broken scaling symmetry. Finally, in conclusion (5) we will outline our results and suggest possible extension of this work.

\section{The effective action for non-BPS Dp-brane in Dk-brane background}

In this section we will analyse the motion of a non-BPS Dp-brane near the stack of coincident and static Dk-branes using the tachyon effective action proposed in [20, 22, 23, 21]. The metric, the dilaton $(\Phi)$, and the R-R field (C) for a system of

\footnotetext{
${ }^{3}$ The form of this symmetry is similar to the symmetry discussed in [31, 32].

${ }^{4}$ We label by symbol $D$ the generator of the scaling symmetry.
} 
$N$ coincident Dk-branes is given by ${ }^{5}$

$$
\begin{array}{r}
g_{\alpha \beta}=H_{k}^{-\frac{1}{2}} \eta_{\alpha \beta}, g_{m n}=H_{k}^{\frac{1}{2}} \delta_{m n},(\alpha, \beta=0,1, \ldots, k, m, n=k+1, \ldots, 9) \\
e^{2 \Phi}=H_{k}^{\frac{3-k}{2}}, C_{0 \ldots k}=H_{k}^{-1}, H_{k}=1+\frac{\lambda}{r^{7-k}}, \lambda=N g_{s} l_{s}^{7-k}
\end{array}
$$

where $H_{k}$ is a harmonic function of $N$ Dk-branes satisfying the Green function equation in the transverse space.

Now let us consider a non BPS Dp-brane with $p<k$ that is inserted in the background (2.1) with its spatial section stretched in directions $\left(x^{1}, \ldots, x^{p}\right)$. We will label the worldvolume of the non-BPS Dp-brane by $\xi^{\mu}, \mu=0, \ldots, p$ and use reparametrization invariance of the worldvolume of the Dp-brane to set $\xi^{\mu}=x^{\mu}$. The position of the D-brane in the transverse directions $\left(x^{p+1}, \ldots, x^{9}\right)$ gives to rise to scalar fields on the worldvolume of D-brane, $\left(X^{p+1}\left(\xi^{\mu}\right), \ldots X^{9}\left(\xi^{\mu}\right)\right)$. We also restrict ourselves to the case of homogeneous modes on the worldvolume of non-BPS Dp-brane. Then the non-BPS Dp-brane effective action ${ }^{6}$ takes the form

$$
S=-\tau_{p} \int d t \sqrt{F} H_{k}^{\frac{k-p-4}{4}} \sqrt{1-\dot{Y}^{i} \dot{Y}^{i}-H_{k} \dot{X}^{m} \dot{X}^{m}-l_{s}^{2} H_{k}^{\frac{1}{2}} F \dot{T}^{2}} \equiv-\int d t \mathcal{L}
$$

where $\tau_{p}$ is a tension of the non-BPS Dp-brane and where $F$ is defined as

$$
F=\frac{1}{1+\frac{T^{2}}{2}} .
$$

From here on we reserve the coordinates $X^{m}$ with $(m=k+1, \ldots, 9)$ for dimensions transverse to Dk-brane and the coordinates $Y^{i}$ with $(i=p+1, \ldots, k)$ for those dimensions transverse to non-BPS Dp-brane, but parallel to the Dk-brane. Finally in (2.2) we have striped off the volume factor of the Dp-brane spatial section $V_{p}$.

To simplify the problem further we use the manifest rotation invariance in transverse $R^{9-k}$ plane. Then we can restrict ourselves to the motion in the $\left(x^{8}, x^{9}\right)$ plane where we introduce the cylindrical coordinates

$$
X^{8}=R \cos \theta, X^{9}=R \sin \theta .
$$

In this paper we will be interested in the region of the field theory space when non-BPS Dp-brane is near the stack of $N$ Dk-branes $\left(\frac{\lambda}{R^{k-7}} \gg 1\right)$ and when the

\footnotetext{
${ }^{5}$ In what follows we consider the background Dk-branes to be static and ignore massive closed string modes. These modes become relevant at $r \ll l_{s}$. But here we assume $l_{s}^{7-k} \ll r^{7-k} \ll$ $N g_{s} l_{s}^{7-k}$. Then the DBI action is valid in this domain and we can study the dynamics of branes in terms of effective action.

${ }^{6}$ This is the form of the tachyon effective action that was proposed in 24, 25].
} 
tachyon is large $\left(T^{2} \gg 1\right)$. In this approximation the non-BPS Dp-brane effective action takes the form

$$
S=-\tau_{p} \sqrt{2} \lambda^{\frac{k-p-4}{4}} \int d t \frac{1}{T R^{\frac{(7-k)(k-p-4)}{4}}} \sqrt{1-\dot{Y}^{i} \dot{Y}^{i}-\frac{\lambda}{R^{7-k}}\left(\dot{R}^{2}+R^{2} \dot{\theta}^{2}\right)-l_{s}^{2} \frac{2 \sqrt{\lambda}}{T^{2} R^{\frac{7-k}{2}}} \dot{T}^{2}} .
$$

Now we come to the main point of this paper, namely to the definition of the scaling transformation. Let us demand that the action (2.5) should be invariant under following transformations

$$
\begin{aligned}
& t^{\prime}=\Gamma^{\alpha} t, T^{\prime}\left(t^{\prime}\right)=\Gamma^{\beta} T(t), R^{\prime}\left(t^{\prime}\right)=\Gamma^{\gamma} R(t), \\
& \theta^{\prime}\left(t^{\prime}\right)=\Gamma^{\delta} \theta(t), Y^{\prime i}\left(t^{\prime}\right)=\Gamma^{\epsilon} Y^{i}(t), \lambda=\Gamma^{\omega} \lambda,
\end{aligned}
$$

where $\Gamma$ is free parameter of this scaling transformation and where $\alpha, \beta, \gamma, \delta, \omega, \epsilon$ will be determined from the requirement that the action (2.5) has to be invariant with respect to (2.6). For example, if we demand that

$$
d t^{\prime} \frac{\lambda^{\prime \frac{k-p-4}{4}}}{T^{\prime} R^{\prime \frac{(7-k)(k-p-4)}{4}}}=d t \frac{\lambda^{\frac{k-p-4}{4}}}{T R^{\frac{(7-k)(k-p-4)}{4}}}
$$

we get

$$
\alpha-\beta-\frac{(7-k)(k-p-4)}{4} \gamma+\frac{(k-p-4)}{4} \omega=0 .
$$

If we proceed in the same way with all other terms in (2.5) we obtain following set of equations

$$
\begin{array}{r}
\omega-2 \alpha=(5-k) \gamma, \\
\omega+2 \delta-2 \alpha=(7-k) \gamma, \\
\omega-4 \alpha=(7-k) \gamma \\
\epsilon=\alpha .
\end{array}
$$

From the first three equations we get

$$
\delta=0, \gamma=-\alpha, \omega=(k-3) \alpha .
$$

Inserting this result into (2.8) we obtain

$$
\beta=\alpha(k-p-3)
$$


The result of this analysis is that all parameters are expressed through $\alpha$. Since different $\alpha$ correspond to different $\Gamma$ we are free to pick one particular value of $\alpha$. If we choose $\alpha=-1$ the scaling transformations take the form

$$
\begin{array}{r}
t^{\prime}=\Gamma^{-1} t, T^{\prime}\left(t^{\prime}\right)=\Gamma^{-(k-p-3)} T(t), R^{\prime}\left(t^{\prime}\right)=\Gamma R(t), \\
\theta^{\prime}\left(t^{\prime}\right)=\theta(t), Y^{\prime i}\left(t^{\prime}\right)=\Gamma^{-1} Y^{i}(t), \lambda^{\prime}=\Gamma^{3-k} \lambda .
\end{array}
$$

As we have claimed in introduction the action (2.5) is generally invariant under (2.12) on condition that parameter $\lambda$ transforms as well ${ }^{7}$. It then follows that the generator of these transformations is not conserved. This can be seen as follows. let us consider following transformations

$$
\begin{array}{r}
t^{\prime}=t+\omega(t) \epsilon, \\
\Phi_{i}^{\prime}\left(t^{\prime}\right)=\Phi_{i}(t)+\Omega_{i}(t) \epsilon \\
m_{b}^{\prime}=m_{b}+\beta_{b}(t) \epsilon,
\end{array}
$$

where $\Phi_{i}$ are dynamical variables and $m_{b}$ are parameters in the action

$$
S=-\int d t \mathcal{L}\left(\Phi_{i}, \dot{\Phi}_{i}, m_{b}\right)
$$

and where we have introduced the infinitisemal parameter $\epsilon \ll 1$. Now we demand that the action (2.14) is invariant under (2.13) in the following sense

$$
\int_{t_{1}}^{t_{2}} d t \mathcal{L}\left(t, \Phi_{i}(t), \frac{d \Phi_{i}}{d t}, m_{b}\right)=\int_{t_{1}^{\prime}}^{t_{2}^{\prime}} d t^{\prime} \mathcal{L}\left(t^{\prime}, \Phi_{i}^{\prime}\left(t^{\prime}\right), \frac{d \Phi_{i}^{\prime}}{d t^{\prime}}, m_{b}^{\prime}\right) .
$$

If we insert (2.13) into the right hand side of this expression and perform the Taylor expansion with respect to $\epsilon$ we get

$$
\int_{t_{1}}^{t_{2}} d t\left[\frac{d}{d t}\left(\omega\left(\mathcal{L}-\dot{\Phi} \frac{\delta \mathcal{L}}{\delta \dot{\Phi}_{i}}\right)+\Omega_{i} \frac{\delta \mathcal{L}}{\delta \dot{\Phi}_{i}}\right)+\left(\Omega_{i}-\omega \dot{\Phi}_{i}\right)\left(\frac{\delta \mathcal{L}}{\delta \Phi_{i}}-\frac{d}{d t}\left(\frac{\delta \mathcal{L}}{\delta \dot{\Phi}_{i}}\right)\right)\right]=-\int_{t_{1}}^{t_{2}} d t \frac{\partial \mathcal{L}}{\partial m_{b}} \beta_{b} .
$$

Now the second term on the left hand side of the previous equation is equal to zero thanks to the equations of motion. Then comparing terms on the left and right side of the upper expression we get

$$
\begin{array}{r}
\frac{d J}{d t}=-\frac{\partial \mathcal{L}}{\partial m_{b}} \beta_{b} \\
J \equiv \omega\left(\mathcal{L}-\dot{\Phi}_{i} \frac{\delta \mathcal{L}}{\delta \dot{\Phi}_{i}}\right)+\Omega_{i} \frac{\delta \mathcal{L}}{\delta \dot{\Phi}_{i}} .
\end{array}
$$

\footnotetext{
${ }^{7}$ One exception is the case of D3-brane background that was extensively studied in [19].
} 
This result explicitly shows that in case when the parameters in Lagrangian have to vary $\left(\beta_{b} \neq 0\right)$ in order the action (2.14) was invariant under (2.13) the generator of given transformation $J$ is not conserved.

Using the general discussion given above we can easily find the equation that determines time evolution of the generator of the transformations (2.12). It is easy to see that their infinitesimal form implies following values of $\omega, \Omega_{i}$ and $m_{b}$

$$
\begin{array}{r}
\omega(t)=-t, \Omega_{T}=-(k-p-3) T(t), \Omega_{R}=R(t), \\
\Omega_{\theta}=0, \Omega_{Y^{i}}=-Y^{i}(t), \beta_{\lambda}=(3-k) \lambda .
\end{array}
$$

Then from (2.17) we get that the generator $J \equiv D$ is equal to

$$
D=t H-(k-p-3) P_{T} T+P_{R} R-P_{i} Y^{i},
$$

where $P_{R}, P_{T}, P_{\theta}, P_{i}$ and $H$ are the canonical momenta that using the action (2.5) take the form

$$
\begin{gathered}
P_{R}=\frac{\delta \mathcal{L}}{\delta \dot{R}}=\frac{\sqrt{2} \tau_{p} \lambda^{\frac{k-p-4}{2}}}{T R^{\frac{(7-k)(k-p-4)}{4}}} \frac{\lambda \dot{R}}{R^{7-k} \sqrt{(\ldots)}}, \\
P_{\theta}=\frac{\delta \mathcal{L}}{\delta \dot{\theta}}=\frac{\sqrt{2} \tau_{p} \lambda^{\frac{k-p-4}{2}}}{T R^{\frac{(7-k)(k-p-4)}{4}}} \frac{\lambda R^{2} \dot{\theta}}{R^{7-k} \sqrt{(\ldots)}}, \\
P_{T}=\frac{\delta \mathcal{L}}{\delta \dot{T}}=\frac{\sqrt{2} \tau_{p} \lambda^{\frac{k-p-4}{2}}}{T R^{\frac{(7-k)(k-p-4)}{4}}} \frac{l_{s}^{2} \sqrt{\lambda} \dot{T}}{T^{2} R^{\frac{7-k}{2}}} \sqrt{(\ldots)} \\
P_{i}=\frac{\delta \mathcal{L}}{\delta \dot{Y}^{i}}=\frac{\sqrt{2} \tau_{p} \lambda^{\frac{k-p-4}{2}}}{T R^{\frac{(7-k)(k-p-4)}{4}}} \frac{\dot{Y}^{i}}{\sqrt{(\ldots)}} .
\end{gathered}
$$

Finally, the Hamiltonian is equal to

$$
H=\sqrt{V^{2}+\frac{P_{R}^{2} R^{7-k}}{\lambda}+\frac{P_{\theta}^{2} R^{7-k}}{\lambda R^{2}}+P_{i}^{2}+\frac{P_{T}^{2} T^{2} R^{\frac{7-k}{2}}}{2 l_{s}^{2} \sqrt{\lambda}}},
$$

where $V^{2}$ is defined as

$$
V^{2}=\tau_{p}^{2} \frac{2}{T^{2}}\left(\frac{\lambda}{R^{7-k}}\right)^{\frac{k-p-4}{2}}
$$

Using (2.21) we obtain following differential equations for $R, \theta, Y^{i}$ and $T$

$$
\begin{gathered}
\dot{R}=\frac{\partial H}{\partial P_{R}}=\frac{P_{R} R^{7-k}}{\lambda E}, \\
\dot{\theta}=\frac{\partial H}{\partial P_{\theta}}=\frac{P_{\theta} R^{7-k}}{\lambda R^{2} E},
\end{gathered}
$$




$$
\begin{gathered}
\dot{T}=\frac{\partial H}{\partial P_{T}}=\frac{T^{2} R^{\frac{7-k}{2}}}{2 l_{s}^{2} \sqrt{\lambda} E} \\
\dot{Y}^{i}=\frac{\partial H}{\partial P_{i}}=\frac{P_{i}}{E}
\end{gathered}
$$

where we have used the fact that Hamiltonian is conserved and equal to energy $E$. It turns out also that it is useful to express the term $\frac{\delta \mathcal{L}}{\delta \lambda}$ as a function of canonical variables

$$
\frac{\delta \mathcal{L}}{\delta \lambda}=-\frac{E}{4 \lambda}\left(-2+\frac{(k-p-2)}{E^{2}}\left(V^{2}+P_{i}^{2}\right)\right)
$$

Finally, in order to simplify the resulting expressions we will consider from now on all momenta $P_{i}$ to be equal to zero. Then the equation that determines time evolution of $D$ takes the form

$$
\frac{d D}{d t}=-\lambda(3-k) \frac{\delta \mathcal{L}}{\delta \lambda}=\frac{(k-3) E}{2}\left(1-\frac{V^{2}}{2 E^{2}}\right) .
$$

It is very difficult to solve this equation in the full generality. On the other hand we see that the term on the right hand side will be time independent if $V^{2}=0$ and the integration of (2.25) is then trivial. As follows from (2.22) this condition can be ensured when $T= \pm \infty$. In the next section we will study this situation in more detail.

\section{Non-BPS Dp-brane in its stable vacuum}

In this section we will consider the situation when the tachyon is static $\left(P_{T}=0\right)$ and reaches its global minimum $(T= \pm \infty)^{8}$. Since now $V^{2}=0$ the equation (2.25) can be easily integrated with the result

$$
D=\frac{E(k-3)}{2} t+D_{0}
$$

or alternatively

$$
D_{0}=t E \frac{(5-k)}{2}+P_{R} R
$$

where $D_{0}$ is the value of the charge $D$ at time $t=0$. This equation allows us to express $P_{R}$ as a function of $R$ and $t$ so that

$$
P_{R}=\frac{1}{R}\left(D_{0}-\frac{(5-k) t E}{2}\right) .
$$

\footnotetext{
${ }^{8}$ More general situations when $P_{T} \neq 0$ will be considered in the next publication.
} 
Then we can easily find the time dependence of $R$ using the first equation in (2.23)

$$
\dot{R}=\frac{P_{R} R^{7-k}}{\lambda E}=\frac{1}{\lambda}\left[\frac{D_{0}}{E}-\frac{(5-k)}{2} t\right] R^{6-k}
$$

that for $k \neq 5$ has the solution

$$
R^{k-5}=\frac{k-5}{\lambda}\left[\frac{D_{0}}{E} t-\frac{(5-k)}{4} t^{2}\right]+C .
$$

The case $k=5$ will be analysed separately. Explicitly, we obtain:

- $\mathrm{k}=6$

In this case the time dependence of $R$ given in (3.5) takes the form

$$
R=\frac{1}{\lambda}\left[\frac{D_{0}}{E} t+\frac{1}{4} t^{2}\right]+R_{0},
$$

where $R_{0}$ is the radial distance of a non-BPS Dp-brane at time $t=0$. Note that $R_{0}$ is related to the initial momentum and to the $D_{0}$ through the relation $D_{0}=P_{R}(0) R_{0}$. Remarkably, even if the tachyon is in its stable minimum $T= \pm \infty$ the dynamics of the radial mode is still nontrivial with the following physical picture: Non-BPS Dp-brane moves from the initial position $R_{i}$, that obeys $\frac{\lambda}{R_{i}} \gg 1$, towards to the stack of $N$ D6-branes until it reaches its turning point ${ }^{9}$ at $t_{T}=-\frac{2 D_{0}}{E}$. Then it moves outwards the stack of $N$ D6-branes until it reaches the region where the approximation $\frac{\lambda}{R} \gg 1$ ceases to be valid. Note that this result is consistent with the general discussion performed in [19].

- $\mathrm{k}=5$

For $k=5$ we obtain from (3.4)

$$
\ln R=\frac{1}{\lambda} \frac{D_{0}}{E} t+\ln C
$$

that implies

$$
R=R_{0} e^{\frac{D_{0}}{\lambda E} t},
$$

where $D_{0}=P_{R}(0) R_{0}, E=\sqrt{\frac{P_{R}^{2}(0) R_{0}^{2}}{\lambda}+\frac{P_{\theta}^{2}}{\lambda}}$. We see that for $D_{0}>0$ the probe Dp-brane leaves the worldvolume of $N$ D5-branes at $t=-\infty$ and moves outwards. After finite time it reaches the region where the approximation of small $R$ ceases to be valid. On the other hand for $D_{0}<0$ we should consider the positive $t$ only. Then Dp-brane starts to move from its distance $R_{0}, \frac{\lambda}{R_{0}} \gg 1$ at time $t=0$ towards to the stack of $N$ D5-brane that it reaches at $t=\infty$.

\footnotetext{
${ }^{9}$ As usual the turning point is the point where the radial velocity of Dp-brane is equal to zero: $\dot{R}=0$.
} 
- $\mathrm{k}<5$

In these cases the solution (3.5) can be written as

$$
\frac{\lambda}{R^{5-k}}=\frac{(5-k)^{2}}{4} t^{2}-(5-k) \frac{D_{0}}{E} t+\frac{\lambda}{R_{0}^{5-k}},
$$

where again $R_{0}$ is the radial position of a Dp-brane at $t=0$ and where $D_{0}$ and $E$ are equal to

$$
D_{0}=P_{R}(0) R_{0}, E=\sqrt{\frac{P_{R}^{2}(0) R_{0}^{7-k}}{\lambda}+\frac{P_{\theta}^{2} R_{0}^{5-k}}{\lambda}} .
$$

Now the physical picture is different from the case of D6 and D5-brane background. Namely, from (3.9) it is easy to see that $R$ blows up for finite $t$ which happens at

$$
t_{ \pm}=\frac{2(5-k)}{(5-k)^{2}}\left[\frac{D_{0}}{E} \pm \sqrt{\frac{D_{0}^{2}}{E^{2}}-\frac{\lambda}{R_{0}^{5-k}}}\right] .
$$

Since by presumption we work in the regime where $\frac{\lambda}{R^{7-k}} \gg 1$ we demand that $R$ is finite for all $t$. This requirement implies that

$$
\frac{D_{0}^{2}}{E^{2}}-\frac{\lambda}{R_{0}^{7-k}}<0 \Rightarrow \frac{P_{\theta}^{2} R_{0}^{3-k}(0)}{P_{R}^{2}}>0
$$

that is always obeyed for $P_{\theta} \neq 0$. In this case we obtain following physical picture. An unstable Dp-brane leaves the stack of $N$ Dk-branes at $t=-\infty$ and reaches its turning point at $t_{T}=\frac{2 D_{0}}{(5-k) E}$ at the distance

$$
R\left(t_{T}\right)=R_{0}\left[1+\frac{P_{R}^{2}(0)}{P_{\theta}^{2}} R_{0}^{2}\right]^{\frac{1}{5-k}}
$$

After the crossing its turning point Dp-brane starts to move towards to the stack of Dk-branes which reaches at the $t=\infty$.

In this section we have studied the dynamics of the non-BPS Dp-brane in the near horizon region of $N$ Dk-branes when the tachyon field reaches its stable minimum. We have shown that in this case there is still nontrivial dynamics present. We mean that the radial mode now describe the collective motion of the gas of highly excited closed strings that arises in the process of the tachyon condensation. We will give more comments to this suggestion in the concussion. 


\section{Dynamics of the BPS Dp-brane in the near horizon region of $N$ Dk-branes and broken scaling symmetry}

This section is devoted to the study of the dynamics of the BPS Dp-brane probe near the stack of $N$ Dk-branes using the scaling symmetry of the Dp-brane effective action. When we again restrict ourselves to the homogeneous fields on the worldvolume of Dp-brane and to the case of the motion in the transverse $\left(x^{8}, x^{9}\right)$ plane then the action for the BPS Dp-brane in the near horizon region of $N$ Dk-branes takes the form

$$
S=-\int d t V \sqrt{1-\dot{Y}^{i} \dot{Y}^{i}-\frac{\lambda}{R^{7-k}}\left(\dot{R}^{2}+R^{2} \dot{\theta}^{2}\right)}=-\int d t \mathcal{L}
$$

where we have striped off the volume factor $V_{p}$ and we have introduced the potential

$$
V=\frac{T_{p} \lambda^{\frac{k-p-4}{4}}}{R^{\frac{(7-k)(k-p-4)}{4}}} .
$$

Note also that now $T_{p}$ is a tension of a BPS Dp-brane that is related to the tension of a non-BPS Dp-brane $\tau_{p}$ as $\tau_{p}=\sqrt{2} T_{p}$. Finally, the meaning of $Y^{i}$ is the same as in the case of non-BPS Dp-brane studied in previous sections.

Let us now demand that the action (4.1) is invariant under following transformations:

$$
t^{\prime}=\Gamma^{\alpha} t, R^{\prime}\left(t^{\prime}\right)=\Gamma^{\gamma} R(t), \theta^{\prime}\left(t^{\prime}\right)=\Gamma^{\delta} \theta(t), \lambda=\Gamma^{\omega} \lambda, Y^{\prime i}\left(t^{\prime}\right)=\Gamma^{\beta} Y^{i}(t)
$$

By comparing the original and transformed action we obtain following set of equations for parameters $\alpha, \delta, \omega, \beta$

$$
\begin{aligned}
\frac{(k-p-4)}{4} \omega+\alpha-\frac{(7-k)(k-p-4)}{4} \gamma & =0, \\
\omega+2 \gamma-(7-k) \gamma-2 \alpha & =0, \\
\omega+2 \gamma-(7-k) \gamma-2 \alpha+2 \delta & =0, \\
\beta-\alpha & =0 .
\end{aligned}
$$

From the last three equations we immediately get that $\delta=0$ and also

$$
\omega+(k-5) \gamma-2 \alpha=0
$$

When we insert this relation into the first equation in (4.4) we obtain the equation

$$
(k-p-2) \omega+[2(k-5)-(7-k)(k-p-4)] \gamma=0
$$


that for $(k-p-2) \neq 0$ has the solution

$$
\omega=\frac{1}{(k-p-2)}[(7-k)(k-p-4)-2(k-5)] \gamma .
$$

For $(k-p-2)=0$ the equation (4.6) implies $\gamma=0$ and (4.5) that $\omega=2 \alpha$. Then for $k=p+2$ and for $\alpha=-1$ the scaling transformations (4.3) take the form

$$
R^{\prime}\left(t^{\prime}\right)=R(t), \lambda^{\prime}=\Gamma^{-2} \lambda, t^{\prime}=\Gamma^{-1} t, Y^{\prime i}\left(t^{\prime}\right)=\Gamma^{-1} Y^{i}(t)
$$

One can now determine the generator of given transformation exactly in the same way as in previous sections with the result

$$
D=t H-P_{i} Y^{i}
$$

whose time evolution is governed by equation

$$
\frac{d D}{d t}=2 \lambda \frac{\delta \mathcal{L}}{\delta \lambda}=E-\frac{P_{i}^{2}}{E}
$$

where we have used that

$$
\frac{\delta \mathcal{L}}{\delta \lambda}=-\frac{E}{4 \lambda}\left(-2+\frac{2 P_{i}^{2}}{E^{2}}+\frac{(k-p-2)}{E^{2}} V^{2}\right) .
$$

On the other hand the explicit time derivative of $D$ given in (4.9) is equal to

$$
\frac{d D}{d t}=H-P_{i} \dot{Y}^{i}=E-\frac{P_{i}^{2}}{E},
$$

as a consequence of the fact that $H$ and $P_{i}$ are conserved. These results then confirm the validity of (4.10). However we also see that the integration of (4.10) is trivial and does not simplify the solution of the equation of motion for $R$ either. Then in order to analyse the dynamics of the radial mode we should use the classical Hamiltonian treatment that was however extensively disused previously in [16, 29.

Let us now consider another possibility when $k-p-4=0$. Now looking at the equations (4.4) we see that the first equation here implies that $\alpha=0$ and consequently $\beta=0$. Then from the second and third equations in (4.4) we obtain

$$
\omega+(k-5) \gamma=0
$$

Since in our convention the radial mode scales as $R \rightarrow \Gamma R$ we take $\gamma=1$. Then we obtain following transformation rules:

$$
R^{\prime}\left(t^{\prime}\right)=\Gamma R(t), t^{\prime}=t, \lambda^{\prime}=\Gamma^{-(k-5)} \lambda
$$


Now it is simple exercise to determine the corresponding generator of given transformations

$$
D=P_{R} R
$$

that obeys the equation

$$
\frac{d D}{d t}=\frac{(k-5) E}{2}\left(1-\frac{P_{i}^{2}}{E^{2}}-\frac{V^{2}}{E^{2}}\right) .
$$

Note that for $(k-p-4)=0$ the potential term $V$ equal to $V=T_{p}$.

Let us now explicitly solve the equation of motion for $R$ using the equation (4.16). Since the Hamiltonian of the probe Dp-brane takes the form

$$
H=\sqrt{V^{2}+\frac{P_{R}^{2} R^{7-k}}{\lambda}+\frac{P_{\theta}^{2} R^{5-k}}{\lambda}+P_{i}^{2}}
$$

the equation of motion for $R$ is equal to

$$
\dot{R}=\frac{\partial H}{\partial P_{R}}=\frac{P_{R} R^{7-k}}{\lambda E} .
$$

Since on the right hand side of the equation (4.16) we have factors that do not depend on time its integration is straightforward and gives

$$
D=P_{R} R=\frac{(k-5) E t}{2}\left(1-\frac{T_{p}^{2}}{E^{2}}\right)+D_{0},
$$

where in order to simplify the notation we have considered the situation when all $P_{i}$ are equal to zero. If we use (4.19) to express $P_{R}$ as function of $t$ and $R$ we get

$$
P_{R}=\frac{1}{R}\left(\frac{(k-5) E t}{2}\left(1-\frac{T_{p}^{2}}{E^{2}}\right)+D_{0}\right) .
$$

Then inserting the upper expression into (4.18) we obtain

$$
d R R^{k-6}=\frac{1}{\lambda}\left(\frac{(k-5) t}{2}\left(1-\frac{T_{p}^{2}}{E^{2}}\right)+\frac{D_{0}}{E}\right) d t
$$

that for $k \neq 5$ has the solution

$$
R^{k-5}=\frac{(k-5)}{\lambda}\left[\frac{(k-5)}{4} t^{2}\left(1-\frac{T_{p}^{2}}{E^{2}}\right)+\frac{D_{0}}{E} t\right]+R_{0}^{k-5} .
$$

Note that for $E=T_{p}$ that corresponds to $P_{R}=P_{\theta}=0$ we have $R=R_{0}$. This fact manifestly demonstrates the supersymmetric nature of the configuration consisting of the Dk-branes $+\mathrm{D}(\mathrm{k}-4)$-branes that do not move. Now we will discuss the solution with $T_{p} \neq E$ in more details. 
- $\mathrm{k}=6$

In this case the solution (4.22) is equal to

$$
R=\frac{1}{\lambda}\left[\frac{1}{4} t^{2}\left(1-\frac{T_{p}^{2}}{E^{2}}\right)+\frac{D_{0}}{E} t\right]+R_{0} .
$$

We see that the probe D2-brane moves initially towards to the stack of $N$ D6branes reaches its turning point at $t_{T}=-\frac{2 D_{0}}{E}\left(1-\frac{T_{p}^{2}}{E^{2}}\right)^{-1}$ and then it moves outwards. Of course this solution is only valid for such a time interval when we have $\frac{\lambda}{R} \gg 1$. Note also that this result is in agreement with the general analysis performed in 29].

- $\mathrm{k}=5$

For $k=5$ the differential equation (4.21) takes the form

$$
\dot{R}=\frac{D}{\lambda E} R
$$

that has the solution

$$
R=R_{0} e^{\frac{D}{\lambda E} t}
$$

We see that the character of the motion of the probe D1-brane depends on the sign of $D=P_{R}(0) R_{0}$. For $D<0$ the probe D1-brane moves towards the stack of D5-branes and reaches it at the asymptotic future. On the other hand the case $D>0$ corresponds to the emission of D1-brane from the worldvolume of $N$ D5-branes at far past $(t=-\infty)$ that then moves outwards and after some time reaches the region where the near horizon approximation breaks down. For $D=0$ the radial coordinate $R$ is constant.

- $\mathrm{k}=4$

In this case (4.22) is equal to

$$
\frac{1}{R}=\frac{1}{\lambda}\left[\frac{t^{2}}{4}\left(1-\frac{T_{p}^{2}}{E^{2}}\right)-\frac{D_{0}}{E} t\right]+\frac{1}{R_{0}}
$$

with the following physical picture. The probe D0-brane leaves the stack of $N$ D4-branes at $t=-\infty$ and moves outwards until it reaches its turning point at $t_{T}=\frac{2 D_{0}}{E}\left(1-\frac{T_{p}^{2}}{E^{2}}\right)^{-1}$. Then it moves back and approaches the worldvolume of the D4-branes at $t=\infty$.

- $\mathbf{k}<4$ In this case one can easily see that there is not any dynamical probe Dp-brane since now $p=k-4<0$. 
As a final case we will consider the situation when $k=6$ and $p=0$. Then from (4.7) we get that $\omega=0$ and from the equations in (4.4) we obtain that $\gamma=2 \alpha$. As a result we obtain following transformation rules for $R, t, Y^{i}$ and $\lambda$

$$
t^{\prime}=\Gamma^{-1} t, R^{\prime}\left(t^{\prime}\right)=\Gamma^{-2} R(t), Y^{\prime i}\left(t^{\prime}\right)=\Gamma^{-1} Y^{i}(t), \lambda^{\prime}=\lambda,
$$

where we have taken $\alpha=-1$. Then the generator of these transformations is

$$
D=t H-P_{R} R-P_{i} Y^{i}
$$

which is, thanks to the fact that $\lambda^{\prime}=\lambda$, conserved. Using (4.28) we can again express $P_{R}$ as a function of $R, H=E$ and $D$

$$
P_{R}=\frac{1}{R}[t E-D]
$$

where we again consider the case when $P_{i}=0$. Inserting this relation to the differential equation for $R$ we get

$$
\dot{R}=\frac{P_{R} R}{\lambda E}=\frac{t E-D}{\lambda E}
$$

that has the solution

$$
R=\frac{1}{2 \lambda} t^{2}-\frac{D}{\lambda E} t+R_{0}
$$

Now we have the following picture of the time dependent dynamics of the probe D0brane in the near horizon region of $N$ D6-branes ${ }^{10}$. The D0-brane starts to move towards to the stack of $N$ D6-brane at same time $t_{i}<0$ that is chosen in such a way to ensure the validity of the near horizon region approach. Then D0-brane reaches its turning point at $t_{T}=\frac{D}{E}$ where the direction of its motion changes and it again moves outwards until it reaches the region where the near horizon approximation breaks down.

\section{Conclusion}

This paper was devoted to the study of the dynamics of the non-BPS and BPS Dpbranes in the near horizon region of the stack of $N$ Dk-branes based on an existence of the broken scaling symmetry of the probe effective action in this background. We have studied the question under which conditions Dp-brane effective action is invariant under this form of the symmetry and we have argued that this generally occurs in case when we allow the scaling parameter $\lambda$ to vary as well. Then we have found the differential equation that determines the time evolution of this generator and we have argued that in the case when the tachyon is sitting in its stable vacuum

\footnotetext{
${ }^{10}$ In order to avoid the coupling of the probe D0-brane to the Ramond-Ramond field we consider the situation when $P_{\theta}=0$. For discussion of the more general case when $P_{\theta} \neq 0$, see [29].
} 
this equation can be easily solved. We mean that it is very interesting that there exists nontrivial dynamics of an unstable Dp-brane even if the tachyon reaches its stable minimum. It is seems to us that this situation is closely related to the emergence of the tachyon matter [33, 34, 35, 36]. Since it was argued in these papers that the tachyon condensation results into the emergence of the gas of very massive closed strings that are confined to the worldvolume of the unstable D-brane [37, 38, 39] then we can presume that the radial mode dynamics captures the collective motion of the gas of these closed strings.

We have also performed an analysis of the probe BPS Dp-brane in the near horizon region of $N$ Dk-branes that was based on an emergence of the scaling symmetry. As in the non-BPS Dp-brane case we have found the generator of this scaling symmetry and the equation that governs its time evolution. Then we have solved the equation of motion of the probe Dp-brane using the known time dependence of $D$.

We mean that extension of this work could be performed in several ways. Firstly, it would be interesting to study the scaling symmetry for situations when the worldvolume fields depend on spatial coordinates as well. Secondly, it would be certainly interesting to study the properties the tachyon matter in general background, espe-

cially it seems very interesting to study the gas of unstable D-branes in the context of the brane gas cosmology. We hope to return to these problems in future.

Acknowledgement This work was supported by the Czech Ministry of Education under Contract No. MSM 0021622409.

\section{References}

[1] A. Sen, "Time and tachyon," Int. J. Mod. Phys. A 18 (2003) 4869 [arXiv:hepth/0209122].

[2] A. Sen, "Tachyon matter," JHEP 0207 (2002) 065 [arXiv:hep-th/0203265].

[3] A. Sen, "Rolling tachyon," JHEP 0204 (2002) 048 [arXiv:hep-th/0203211].

[4] A. Sen, "Tachyon dynamics in open string theory," arXiv:hep-th/0410103.

[5] D. Kutasov, "D-brane dynamics near NS5-branes," arXiv:hep-th/0405058.

[6] Y. Nakayama, K. L. Panigrahi, S. J. Rey and H. Takayanagi, "Rolling down the throat in NS5-brane background: The case of electrified D-brane," arXiv:hep-th/0412038.

[7] B. Chen, M. Li and B. Sun, "Dbrane near NS5-branes: With electromagnetic field," arXiv:hep-th/0412022.

[8] S. Thomas and J. Ward, "D-brane dynamics and NS5 rings," arXiv:hep-th/0411130.

[9] D. Bak, S. J. Rey and H. U. Yee, "Exactly soluble dynamics of $(p, q)$ string near macroscopic fundamental strings," arXiv:hep-th/0411099. 
[10] J. Kluson, "Non-BPS Dp-brane in the background of NS5-branes on transverse $R^{* * 3}$ $x S^{* *} 1, "$ arXiv:hep-th/0411014.

[11] J. Kluson, “Non-BPS D-brane near NS5-branes,” JHEP 0411, 013 (2004) [arXiv:hepth/0409298].

[12] O. Saremi, L. Kofman and A. W. Peet, "Folding branes," arXiv:hep-th/0409092.

[13] D. Kutasov, "A geometric interpretation of the open string tachyon," arXiv:hepth/0408073.

[14] D. A. Sahakyan, "Comments on D-brane dynamics near NS5-branes," JHEP 0410, 008 (2004) [arXiv:hep-th/0408070].

[15] A. Ghodsi and A. E. Mosaffa, "D-brane dynamics in RR deformation of NS5-branes background and tachyon cosmology," arXiv:hep-th/0408015.

[16] K. L. Panigrahi, "D-brane dynamics in Dp-brane background," Phys. Lett. B 601, 64 (2004) [arXiv:hep-th/0407134].

[17] H. Yavartanoo, "Cosmological solution from D-brane motion in NS5-branes background," arXiv:hep-th/0407079.

[18] Y. Nakayama, Y. Sugawara and H. Takayanagi, "Boundary states for the rolling Dbranes in NS5 background," JHEP 0407, 020 (2004) [arXiv:hep-th/0406173].

[19] J. Kluson, "Non-BPS Dp-brane in Dk-brane background," arXiv:hep-th/0501010.

[20] A. Sen, "Supersymmetric world-volume action for non-BPS D-branes," JHEP 9910 (1999) 008 [arXiv:hep-th/9909062].

[21] J. Kluson, "Proposal for non-BPS D-brane action," Phys. Rev. D 62 (2000) 126003 [arXiv:hep-th/0004106].

[22] E. A. Bergshoeff, M. de Roo, T. C. de Wit, E. Eyras and S. Panda, "T-duality and actions for non-BPS D-branes," JHEP 0005 (2000) 009 [arXiv:hep-th/0003221].

[23] M. R. Garousi, "Tachyon couplings on non-BPS D-branes and Dirac-Born-Infeld action," Nucl. Phys. B 584 (2000) 284 [arXiv:hep-th/0003122].

[24] D. Kutasov and V. Niarchos, "Tachyon effective actions in open string theory," Nucl. Phys. B 666 (2003) 56 [arXiv:hep-th/0304045].

[25] V. Niarchos, "Notes on tachyon effective actions and Veneziano amplitudes," Phys. Rev. D 69 (2004) 106009 [arXiv:hep-th/0401066].

[26] A. Fotopoulos and A. A. Tseytlin, "On open superstring partition function in inhomogeneous rolling tachyon background," JHEP 0312, 025 (2003) [arXiv:hep-th/0310253].

[27] A. Sen, "Moduli space of unstable D-branes on a circle of critical radius," JHEP 0403 (2004) 070 [arXiv:hep-th/0312003]. 
[28] C. P. Burgess, P. Martineau, F. Quevedo and R. Rabadan, "Branonium," JHEP 0306 (2003) 037 [arXiv:hep-th/0303170].

[29] C. P. Burgess, N. E. Grandi, F. Quevedo and R. Rabadan, "D-brane chemistry," JHEP 0401 (2004) 067 [arXiv:hep-th/0310010].

[30] A. Sen, "Dirac-Born-Infeld action on the tachyon kink and vortex," Phys. Rev. D 68 (2003) 066008 [arXiv:hep-th/0303057].

[31] A. Jevicki, Y. Kazama and T. Yoneya, "Generalized conformal symmetry in D-brane matrix models,” Phys. Rev. D 59 (1999) 066001 [arXiv:hep-th/9810146].

[32] A. Jevicki and T. Yoneya, "Space-time uncertainty principle and conformal symmetry in D-particle dynamics," Nucl. Phys. B 535 (1998) 335 [arXiv:hep-th/9805069].

[33] G. W. Gibbons, K. Hori and P. Yi, "String fluid from unstable D-branes," Nucl. Phys. B 596, 136 (2001) [arXiv:hep-th/0009061].

[34] A. Sen, "Fundamental strings in open string theory at the tachyonic vacuum," J. Math. Phys. 42, 2844 (2001) [arXiv:hep-th/0010240].

[35] A. Sen, "Open and closed strings from unstable D-branes," Phys. Rev. D 68, 106003 (2003) [arXiv:hep-th/0305011].

[36] O. K. Kwon and P. Yi, "String fluid, tachyon matter, and domain walls," JHEP 0309 (2003) 003 [arXiv:hep-th/0305229].

[37] A. Sen, "Open-closed duality: Lessons from matrix model," Mod. Phys. Lett. A 19 (2004) 841 [arXiv:hep-th/0308068].

[38] A. Sen, "Open-closed duality at tree level," Phys. Rev. Lett. 91 (2003) 181601 [arXiv:hep-th/0306137].

[39] N. Lambert, H. Liu and J. Maldacena, "Closed strings from decaying D-branes," arXiv:hep-th/0303139. 